\title{
LATTICES OF PSEUDOVARIETIES
}

\author{
P. AGLIANO and J. B. NATION
}

(Received 11 March 1987)

Communicated by T. E. Hall

\begin{abstract}
We consider the lattice of pseudovarieties contained in a given pseudovariety $P$. It is shown that if the lattice $L$ of subpseudovarieties of $P$ has finite height, then $L$ is isomorphic to the lattice of subvarieties of a locally finite variety. Thus not every finite lattice is isomorphic to a lattice of subpseudovarieties. Moreover, the lattice of subpseudovarieties of $P$ satisfies every positive universal sentence holding in all the lattices of subvarieties of varieties $V(A)$ generated by algebras $A \in P$.
\end{abstract}

1980 Mathematics subject classification (Amer. Math. Soc.): 08 B 15, 08 C 99.

A pseudovariety $P$ is a class of finite algebras closed under the formation of homomorphic images, subalgebras and finite direct products: in symbols $\mathbf{H S P}_{\mathbf{f i n}}(P)$ $=P$. This concept has been useful in many investigations, particularly in the study of various classes of finite semigroups and monoids; see [2], [3], [7], [8] and, for a more general approach, [5].

We will consider the lattice of subpseudovarieties of a given pseudovariety $P$. We will show that several recent results about the lattice of subvarieties of a variety have analogs for pseudovarieties.

This investigation originated in a series of discussions between the second author, Kathy Johnston and T. E. Hall at Monash University in August 1986, which produced a direct proof of Corollary 2.6. Hall and Johnston were at tht time working on pseudovarieties of inverse semigroups [8], and Section 5 of that paper contains some interesting results related to the ones herein. C. J. Ash

The second author was supported in part by NSF Grant MCS 83-01087.

(C) 1989 Australian Mathematical Society 0263-6115/89 \$A2.00+0.00 
has also generalized Corollary 2.6 in a rather different direction (see $[8$, Remark 5.5]).

\section{Preliminaries and forbidden lattices}

If $K$ is a class of algebras (of the same type), $\mathrm{V}(K)$ will denote the variety generated by $K$; if $Q$ is a class of finite algebras $\mathbf{P}(Q)=\mathbf{H S P}_{\mathbf{f i n}}(Q)$ will denote the pseudovariety generated by $Q$.

It is clear that, if $V$ is a variety, the class consisting of the finite algebras in $V$ is a pseudovariety. We will denote this class by $V_{\mathbf{f n}}$ or $V \cap$ Fin. On the other hand it is easy to produce examples of pseudovarieties that are not of this kind, for example

(a) the pseudovariety of finite Abelian groups of square - free exponent,

(b) the pseudovariety of finite semigroups satisfying $x^{n}=x^{n+1}$ for some $n$,

(c) the pseudovariety $\mathscr{F}$ where

$$
\mathscr{F}=\left\{F_{1} \times \cdots \times F_{n}: F_{i} \text { is a finite field, } \operatorname{char} F_{i}=p \text { for all } i\right\} .
$$

(d) the pseudovariety of all finite lattices satisfying $S D_{\wedge}$.

The following lemma (whose proof is straightforward) will be used repeatedly. We recall that a variety $V$ is locally finite if all finitely generated algebras in $V$ are finite.

LEMMA 1.1. Let $V$ be a locally finite variety and $A$ a finite algebra in $V$. Suppose there exist a $B \in V$, a family $\left\{C_{\alpha}\right\}_{\alpha<\beta}$ of algebras in $V$ and a surjective homomorphism $h$ such that

$$
A \leftarrow{ }_{h} B \leq \prod\left(C_{\alpha}: \alpha<\beta\right) .
$$

Then there exist finitely many $\alpha_{1}, \ldots, \alpha_{n}$ such that

$$
A \underset{h}{\leftarrow} B^{\prime} \leq C_{\alpha_{1}} \times \cdots \times C_{\alpha_{n}} .
$$

The following lemma can be found in [1], [6] and [11] and was implicitly stated in [7] for monoids.

LEMMA 1.2. A class of finite algebras $P$ is a pseudovariety if and only if there exists a directed union of locally finite varieties such that

$$
P=\bigcup\left(V_{i}: i \in I\right) \cap \text { Fin. }
$$

The proof is straightforward, by taking

$$
I=\{S \subseteq P: S \text { is finite }\}
$$


and noting that by Lemma $1.1, \mathrm{~V}(S)$ is locally finite for all $S \in I$. If $V$ is a variety let $L_{\mathbf{v}}(V)$ be the lattice of subvarieties of $V$, and if $P$ is a pseudovariety let $\mathrm{L}_{\mathbf{p v}}(P)$ be the set of pseudovarieties contained in $P$. It is a routine exercise to prove that $\mathrm{L}_{\mathrm{pv}}(P)$ is indeed an algebraic lattice under inclusion, with HSP $_{\mathbf{f n}}$ being the associated closure operator.

It is not hard to see that the lattices $L_{v}$ and $L_{p v}$ can be quite different even for the same variety of algebras. Let $A b$ be the variety of abelian groups. Then $\mathbf{L}_{\mathbf{v}}(A b)$ is isomorphic to the lattice $\mathbf{N}$ of the positive integers under division with the largest element adjoined, while $\mathbf{L}_{\mathrm{pv}}\left(A b_{\text {fin }}\right)$ is isomorphic to the ideal lattice of $\mathbf{N}$.

We recall that if $L$ is a lattice, the height $h(a)$ of $a \in L$ is the length of the shortest maximal chain in $a / 0$; we say that $P$ is a pseudovariety of finite height if $P$ has finite height in $\mathbf{L}_{\mathbf{p v}}(P)$.

LEMMA 1.3. If $P$ is a pseudovariety of finite height, then $P$ is generated by finitely many finite algebras.

Proof. We induct on the height of $P$. If $h(P)=0$, then $P$ is the trivial pseudovariety generated by a one-element algebra. Assume the statement true for any height $<n$ and let $h(P)=n$. Then $P$ covers $Q$, where $h(Q)<n$ and $Q$ is generated by finitely many finite algebras. Let $A \in Q-P$; then $P<P(Q \cup\{A\}) \leq P$, and since $P$ covers $Q$ we have $P=P(Q \cup\{A\})$. Then $P$ itself is finitely generated, and the lemma is proved.

The next lemma connects pseudovarieties and locally finite varieties.

LEMMA 1.4. Let $V$ be locally finite. Consider the maps

$$
\varphi: \mathbf{L}_{\mathbf{v}}(V) \rightarrow \mathbf{L}_{\mathbf{p v}}\left(V_{\text {fin }}\right)
$$

via $\varphi(U)=U \cap$ Fin, and $\psi: \mathbf{L}_{\mathbf{p v}}\left(V_{\text {fin }}\right) \rightarrow \mathbf{L}_{\mathbf{v}}(V)$ via $\psi(Q)=\mathbf{V}(Q)$. Then

(i) for all pseudovarieties $Q \leq V_{\text {fin }}, Q=\varphi \psi(Q)=\mathrm{V}(Q) \cap \mathrm{Fin}$,

(ii) for all varieties $U \leq V, U=\psi \varphi(U)=\mathbf{V}(U \cap \mathrm{Fin})$,

(iii) $\varphi$ and $\psi$ are lattice isomorphisms.

ProOF. (i) Clearly $Q \subseteq V(Q) \cap$ Fin. If $A$ is a finite algebra in $V(Q)$, then there exist an algebra $B \in V(Q)$, a family $\left\{C_{\alpha}\right\}_{\alpha<\beta}$ of algebras in $Q$ and a surjective homomorphism $h$ such that

$$
A \stackrel{h}{\longleftarrow} B \leq \prod\left(C_{\alpha}: \alpha<\beta\right) .
$$

Since $V$ it is locally finite and $A$ is finite, it can be assumed by Lemma 1.1 that $\beta$ is finite and all the $C_{\alpha}$ are finite. Then $A \in \mathbf{H S P}_{\mathbf{f n}}(Q)=Q$ and we are done.

(ii) is obvious since any $U \leq V$ is itself locally finite, and is therefore generated by its finite members. 
(iii) follows from (i) and (ii), since a one-to-one map $f$ of a lattice $L$ onto a lattice $M$ is an isomorphism if and only if $f$ and its inverse are order-preserving.

The main theorem of this section is now easy to prove.

THEOREM 1.5. If $P$ is a pseudovariety of finite height, then the lattice of pseudovarieties $\mathbf{L}_{\mathrm{pv}}(P)$ is isomorphic to the lattice of subvarieties $\mathbf{L}_{\mathbf{v}}(\mathbf{V}(P))$.

ProOF. By Lemma 1.3, $P$ is generated by finitely many finite algebras; hence $\mathrm{V}(P)$ is locally finite. So Lemma 1.4 applies, and we can conclude that $\mathbf{L}_{\mathbf{p v}}(P) \cong \mathbf{L}_{\mathbf{v}}(\mathbf{V}(P))$.

The following corollary is a direct consequence of the theorem.

COROLLARY 1.6. If $L$ is a lattice of finite height, forbidden as lattice of subvarieties for a locally finite variety, then it is also forbidden as $\mathbf{L}_{\mathbf{p v}}(P)$ for any pseudovariety $P$.

A large class of lattices forbidden for locally finite varieties (the so-called "tight" lattices) can be found in Mckenzie's paper [10].

\section{A representation theorem}

The results of the previous section lead to the question: what properties of $\mathbf{L}_{\mathbf{v}}(V)$ are inherited by $\mathbf{L}_{\mathbf{p v}}\left(V_{\mathrm{fin}}\right)$ (not assuming local finiteness)? This section provides some answers to this question.

THEOREM 2.1. Let $P$ be a pseudovariety and for $A \in P$ let $\mathbf{L}_{\mathbf{A}}=\mathbf{L}_{\mathbf{v}}(\mathbf{V}(A))$. If $\mathscr{K}(P)=\left\{L_{\mathbf{A}}: A \in P\right\}$, then

$$
\mathbf{L}_{\mathbf{p v}}(P) \in \mathbf{H S P}_{\mathbf{u}}\{\mathscr{K}(P)\} .
$$

We will first show that $\mathbf{L}_{\mathrm{pv}}(P)$ is in the variety generated by $\mathscr{K}(P)$. Let $M$ be the direct product $\Pi\left(\mathrm{L}_{\mathbf{A}}: A \in P\right)$, where it is understood that we take an algebra from each isomorphism class. Let

$$
S=\left\{x \in M: A \in \mathrm{V}(B) \text { implies } x_{A} \leq x_{B}\right\} .
$$

Clearly $S$ is a sublattice of $M$. Define $\gamma \subseteq S^{2}$ by setting $(x, y) \in \gamma$ if

(*) $\forall A, C \in P, C \in x_{A}$ implies there exists $B \in P$ such that $A \in \mathbf{V}(B)$ and $C \in y_{B}$ and

(**) $\forall A, C \in P, C \in y_{A}$ implies there exists $B^{\prime} \in P$ such that $A \in \mathrm{V}\left(B^{\prime}\right)$ and $C \in x_{B^{\prime}}$.

We would like to prove that $\gamma \in \operatorname{Con} S$. The proof uses the following straightforward lemma. 
LEMMA 2.2. If $V$ is a locally finite variety, $U, W \leq V$ and $C$ is a finite algebra in $U \vee W$, then there are finite algebras $D \in U$ and $E \in W$ such that $C \in \mathbf{H S}(D \times E)$.

LEMMA 2.3. $\gamma \in \operatorname{Con} S$.

Proof. It is not hard to check that $\gamma$ is an equivalence relation on $S$. Assume now $z \in S, x \gamma y$, and $C \in x_{A} \wedge z_{A}$. By (*) there is a $B \in P$ such that $C \in y_{B}$ and $A \in \mathrm{V}(B)$, whence $C \in z_{B} \geq z_{A}$. Thus $C \in y_{B} \wedge z_{B}$ which proves (*); (**) is similar so we can conclude that $x \wedge z \gamma y \wedge z$.

If $C \in x_{A} \vee z_{A}$ then, by Lemma 2.2, $C \in \mathbf{H S}(D \times E)$ for finite algebras in $x_{A}$ and $z_{A}$ respectively. Because $D \in x_{A},(*)$ yields a $B \in P$ with $A \in \mathrm{V}(P)$ and $D \in y_{B}$. Since $z \in S$ and $A \in \mathrm{V}(B)$, it follows that $E \in z_{B}$ and $C \in y_{B} \vee z_{B}$. The proof of $(* *)$ is similar, so $x \vee z \gamma y \vee z$.

Define a function $\varphi: \mathbf{L}_{\mathbf{p v}}(P) \rightarrow S$ by setting $\varphi_{A}(Q)=\mathbf{V}(Q \cap \mathbf{V}(A))$; it is trivial to see that indeed $\varphi(Q) \in S$ for all $Q \leq P$.

LEMMA 2.4. For all $Q, R \leq P$

(i) $\varphi(Q \cap R)=\varphi(Q) \cap \varphi(R)$,

(ii) $\varphi(Q) \vee \varphi(R) \leq \varphi(Q \vee R)$,

(iii) $\varphi(Q \vee R) \gamma \varphi(Q) \vee \varphi(R)$,

(iv) if $Q \not R R$ then $(\varphi(Q), \varphi(R)) \notin \gamma$.

ProOF. (i) and (ii) are immediate. For (iii), assume that $C$ is finite and $C \in \varphi_{A}(Q \vee R)=\mathrm{V}((Q \vee R) \cap \mathrm{V}(A))$. By Lemma 1.1, $C \in(Q \vee R) \cap \mathrm{V}(A)$, so by Lemma 2.2 there are $D \in Q, E \in R$ with $C \in \mathbf{H S}(D \times E)$. Let $B=D \times E \times A$; then $A \in \mathbf{V}(B)$ and $C \in(Q \cap \mathbf{V}(B)) \vee(R \cap \mathbf{V}(B))$, so $C \in \varphi_{B}(Q) \vee \varphi_{B}(R)$. Thus (*) is satisfied and (**) follows at once from (ii); hence (iii) is proved.

For (iv) take $C \in Q-R$. If $C \in \mathrm{V}(B)$, then by Lemma 1.1,

$$
C \in \mathrm{V}(Q \cap \mathrm{V}(B))-\mathrm{V}(R \cap \mathrm{V}(B)) .
$$

Since $C \notin \varphi_{B}(R)$ for all $B$, we have $(\varphi(Q), \varphi(R)) \notin \gamma$.

We can summarize all we have gotten so far by

THEOREM 2.5. If $P$ is a pseudovariety then $\mathbf{L}_{\mathbf{p v}}(P) \in \operatorname{HSP}\{\mathscr{K}(P)\}$; in fact $\mathrm{L}_{\mathrm{pv}}(P)$ is embedded into $S / \gamma$ via the function $\varphi$ described above.

COROLLARY 2.6. For any lattice equation $\varepsilon$, if $\mathbf{L}_{\mathbf{v}}(V)$ satisfies $\varepsilon$ then $\mathrm{L}_{\mathrm{pv}}\left(V_{\mathrm{fn}}\right)$ satisfies $\varepsilon$.

COROLLARY 2.7. If $\mathrm{L}_{\mathbf{v}}(\mathrm{V}(P))$ satisfies $S D_{\wedge}$ then so does $\mathbf{L}_{\mathrm{pv}}(P)$. 
Proof. If $Q \wedge R_{1}=Q \wedge R_{2}$, then for all $A \in P$

$$
\mathbf{V}\left(Q \wedge R_{1} \cap \mathbf{V}(A)\right)=\mathbf{V}\left(Q \wedge R_{2} \cap \mathbf{V}(A)\right)
$$

implying

$$
\mathbf{V}(Q \cap \mathbf{V}(A)) \wedge \mathbf{V}\left(R_{1} \cap \mathbf{V}(A)\right)=\mathbf{V}(Q \cap \mathbf{V}(A)) \wedge \mathbf{V}\left(R_{2} \cap \mathbf{V}(A)\right)
$$

Since $\mathbf{L}_{\mathbf{v}}(\mathbf{V}(P))$ satisfies $S D_{\wedge}$,

$\mathbf{V}(Q \cap \mathbf{V}(A)) \wedge \mathbf{V}\left(R_{1} \cap \mathbf{V}(A)\right)=\mathbf{V}(Q \cap \mathbf{V}(A)) \wedge\left[\mathbf{V}\left(R_{1} \cap \mathbf{V}(A)\right) \vee \mathbf{V}\left(R_{2} \cap \mathbf{V}(A)\right)\right]$.

Hence

$$
\begin{aligned}
\varphi_{A}\left(Q \wedge R_{1}\right) & =\varphi_{A}(Q) \wedge\left[\varphi_{A}\left(R_{1}\right) \vee_{A}\left(R_{2}\right)\right] \\
& =\varphi_{A}(Q) \wedge\left[\varphi_{A}\left(R_{1}\right) \vee \varphi\left(R_{2}\right)\right]_{A}
\end{aligned}
$$

so, by Lemma 2.4(iii)

$$
\varphi\left(Q \wedge R_{1}\right)=\varphi(Q) \wedge\left[\varphi\left(R_{1}\right) \vee \varphi\left(R_{2}\right)\right] \gamma \varphi(Q) \wedge \varphi\left(R_{1} \wedge R_{2}\right) .
$$

By Theorem 2.5, $Q \wedge R_{1}=Q \wedge\left(R_{1} \vee R_{2}\right)$ and $\mathrm{L}_{\mathrm{pv}}(P)$ satisfies $S D_{\wedge}$.

There is no similar argument for $S D_{\vee}$, and we conjecture that $S D_{\vee}$ and Lampe's condition [9], which is similar, are not preserved by $\mathbf{L}_{\mathbf{p v}}\left(V_{\mathrm{fin}}\right)$.

We can refine Theorem 2.4, to obtain $\mathrm{L}_{\mathrm{pv}}(P) \in \mathbf{H S P}_{\mathbf{u}}\{\mathscr{K}(P)\}$, by the following general argument.

Let $\mathscr{A}=\left(A_{i}\right)_{i \in I}$ be a family of algebras, $N=\prod\left(A_{i}: i \in I\right), S \leq N$ and $\gamma \in$ Con $S$. Let $\mathscr{U}$ be an ultrafilter on $I$, and let $\mu$ be the induced congruence on $N$, that is $x \mu y$ if $\left\{i: x_{i}=y_{i}\right\} \in \mathscr{U}$. Let $T=\{t \in N$ : there exists $x \in S$ with $t \mu x\}$. Note that $T$ is a subalgebra of $N$. Define $\rho \subseteq T^{2}$ by $\left(t, t^{\prime}\right) \in \rho$ if there are $x, x^{\prime} \in S$ with $t \mu x \gamma x^{\prime} \mu t^{\prime}$.

LEMMA 2.8. If $\mu \cap S^{2} \subseteq \gamma$ then $\rho \in \operatorname{Con} T$ and $S / \gamma \cong T / \rho$. Consequently $S / \gamma \in \mathbf{H S P}_{\mathbf{u}}\{\mathscr{A}\}$.

ProOF. $\rho$ is clearly reflexive and symmetric. If $t \rho t^{\prime} \rho t^{\prime \prime}$, then there are $x, x^{\prime}, y^{\prime}, y^{\prime \prime} \in S$ with $t \mu x \gamma x^{\prime} \mu t^{\prime} \mu y^{\prime} \gamma y^{\prime \prime} \mu t^{\prime \prime}$. But then $x^{\prime} \mu y^{\prime}$, so by hypothesis $x^{\prime} \gamma y^{\prime}$. Hence $x \gamma x^{\prime \prime}$ and $t \rho t^{\prime \prime}$. Thus $\rho$ is transitive. This relation clearly respects operations, so that $\rho \in \operatorname{Con} T$.

It is an easy exercise to prove that $\psi: S / \gamma \rightarrow T / \rho$ defined by $\psi(x / \gamma)=x / \rho$ is an isomorphism. Since $\mu \cap T^{2} \subseteq \rho$, we have $T / \rho \in \mathbf{H S P}_{\mathbf{u}}(\{\mathscr{A}\})$.

We want to apply Lemma 2.8 to the specific $S$ and $\gamma$ defined earlier in this section. For $B \in P$, define $\mathscr{S}(B)=\{A \in P: B \in \mathrm{V}(A)\}$. Note that $\{\mathscr{S}(B): B \in$ $P\}$ is a filterbase: $\mathscr{S}(B) \neq \varnothing$, and if $B, C \in P$ then $\mathscr{S}(B) \cap \mathscr{S}(C) \supseteq \mathscr{S}(D)$, where $D=B \times C$. Let $\mathscr{U}$ be any ultrafilter containing this filterbase, and let $\mu$ be the congruence on $M$ associated to $\mathscr{U}$. We need the following facts. 
LemMa 2.9. (i) For all $E \in \mathscr{U}, B \in P$ implies $E \cap \mathscr{S}(B) \neq \varnothing$. (ii) $\mu \cap S^{2} \subseteq \gamma$.

Proof. (i) is trivial from the maximality of $\mathscr{U}$. For (ii), let $(x, y) \in \mu \cap S^{2}$; then $\left\{C: x_{C}=y_{C}\right\} \in \mathscr{U}$. Fix $A \in P$. By (i), $\left\{C: x_{C}=y_{C}\right\} \cap \mathscr{S}(A) \neq \varnothing$ so there is a $B$ such that $A \in \mathrm{V}(B)$ and $x_{B}=y_{B}$. If $D \in x_{A}$ then $x_{A} \leq x_{B}=y_{B}$ since $x \in S$, so $D \in y_{B}$. If $D \in y_{A}$, then $y_{A} \leq y_{B}=x_{B}$, so $D \in x_{B}$. Hence $x \gamma y$ and (ii) is proved.

Lemma 2.9 (ii) allows us to apply Lemma 2.8 to our situation, yielding $S / \gamma \in$ $\operatorname{HSP}_{\mathbf{u}}(\mathscr{K}(P))$. Since $\mathbf{L}_{\mathbf{p v}}(P)$ is isomorphic to a sublattice of $S / \gamma$, we obtain $\mathbf{L}_{\mathbf{p v}}(P) \in \mathbf{H S P}_{\mathbf{u}}\{\mathscr{K}(P)\}$, which is the claim of Theorem 2.1.

COROLLARY 2.10. Any positive universal sentence holding in $\mathrm{L}_{\mathbf{v}}(V)$ is also satisfied by $\mathbf{L}_{\mathbf{p v}}\left(V_{\mathrm{fin}}\right)$.

\section{References}

[1] C. J. Ash, 'Pseudovarieties, generalized varieties and similarly described classes', J. Algebra 92 (1985), 104-115.

[2] J. Almeida, 'Power pseudovarieties of semigroups. I, II', Semigroup Forum 33 (1986), $357-373 ; 375-390$.

[3] J. Almeida, 'The algebra of implicit operations', preprint.

[4] K. A. Baker and A. W. Hales, 'From a lattice to its ideal lattice', Algebra Universalis 4 (1974), 250-258.

[5] J. T. Baldwin and J. Berman, 'Varieties and finite closure conditions', Collog. Math. 35 (1976), 15-20.

[6] B. Banaschewski, 'The Birkhoff Theorem for varieties of finite algebras', Algebra Universalis 17 (1983), 360-368.

[7] S. Eilenberg and M. P. Schützenberger, 'On pseudovarieties', Adv. in Math. 19 (1976), 413-418.

[8] T. E. Hall and K. G. Johnston, 'The lattice of pseudovarieties of inverse semigroups', Pacific J. Math., to appear.

[9] W. A. Lampe, 'A property of the lattice of equational theories', Algebra Universalis 23 (1986), 61-69.

[10] R. McKenzie, 'Finite forbidden lattices', Proceedings of the Universal Algebra and Lattice Theory Conference, Puebla 1982 (Lecture Notes in Math., vol. 1004, Springer-Verlag, pp. 176-205).

[11] J. Reiterman, 'The Birkhoff theorem for finite algebras', Algebra Universalis 14 (1982), 1-10.

Department of Mathematics

University of Hawaii at Manoa

Honolulu

Hawaii 96822 